\title{
International Neuroblastoma Risk Group Classification System
}

National Cancer Institute

\section{Source}

National Cancer Institute. International Neuroblastoma Risk Group Classification System. NCI Thesaurus. Code C102563.

A classification system developed to establish a consensus approach for pretreatment risk stratification of neuroblastomas. 